Selcuk Journal of Agriculture and Food Sciences

http://sjafs.selcuk.edu.tr/sjafs/index

Research Article

\section{SJAFS}

(2021) 35 (1), 49-55

e-ISSN: 2458-8377

DOI:10.15316/SJAFS. 2020.228

\title{
Efficacy of Neem Leaf (Azadirachta indica), Bitter Leaf (Vernonia amygdalina) and Pawpaw Leaf (Carica papaya) Powder in the Control of Callosobruchus maculatus in Stored Cowpea
}

\author{
Aminat Arinola SOLIHU ${ }^{1 *}$, (DOmotayo Robert UDDIN II ${ }^{1}$, iD Samuel Femi BABATUNDE ${ }^{1}$, \\ Lukman Idowu GAMBARI ${ }^{2}$ \\ ${ }^{1}$ Department of Crop Protection, Faculty of Agriculture, University of Ilorin, Ilorin, Nigeria. \\ ${ }^{2}$ Department of Crop Production, College of Agronomy, Federal University of Agriculture, Makurdi, Nigeria
}

\begin{tabular}{l}
\hline ARTICLE INFO \\
\hline Article history: \\
Received date: 09.01 .2021 \\
Accepted date: 31.03 .2021 \\
\hline Edited by: \\
Murat KARACA; Selcuk University, \\
Turkey
\end{tabular}

Keywords:

Cowpea

Vernoniaamygdalina

Azadirachta indica

Carica papaya

Callosobruchus maculatus

Pest management

\begin{abstract}
A laboratory experiment was carried out to investigate the insecticidal properties of Azadirachta indica (Neem), Vernonia amygdalina (Bitter Leaf) and Carica papaya (Pawpaw) leaf powders against Callosobruchus maculatusin stored cowpea. The experiment was carried out using a completely randomized design. The treatments were applied as single and mixed applications at the following rates-3g, $6 \mathrm{~g}$ and $9 \mathrm{~g}$. Each treatment were replicated 4 times making a total of 92 experimental units. Each treatment was applied to $100 \mathrm{~g}$. Data collected include adult mortality, larval and pupa emergence, grain damage, weight loss and $F_{1}$ progeny emergence. The data collected were subjected to analysis of variance (AVONA) at 5\% probability level. The results indicated that, there were significantly differences between plant products treatments and the synthetic treatment over the control throughout the period of the experiment ( 1 to 8 weeks). However, the various treatment of the plant products used for the experiment proved to be effective in controlling C. maculatus of stored cowpea. However, Cypermethrin dust at $0.6 \mathrm{~g} / 100 \mathrm{~g}$ of cowpea was the most effective in controlling grain damage while pawpaw leaf powder and Bitter Leaf powder proved to be most effective in controlling grain damage among the natural botanicals, adult mortality and number of eggs laid by $C$. maculatus on the stored grains. The result clearly indicated the potential values of using plants extracts as complimentary to chemicals pesticides in controlling C. maculatus on cowpea grains.
\end{abstract}

\section{Introduction}

Cowpea (Vignaunguiculata L. Walp) is one of the most ancient crops known to humankind. Its origin and subsequent domestication is associated with pearl millet and sorghum in West Africa Musa et al. (2009). The cowpea was first domesticated in Africa between 1700 to 1500 before the Current Era (Singh, 2014) and all cultivated varieties grown in the world today originated from East and West Africa Xiong et al. (2016). Cowpea seed pods and leaves are consumed in fresh form as green vegetables in some African Countries (Ghaly and Alkoaik, 2010), while the rest of the cowpea plant after the pods have been harvested serves as a nutritious fodder for livestock (Abebe et al., 2005) and also a source of cash income (Dugje et al., 2009). The nutritive value of cowpea makes it an extremely important protein source to vegetarian and people who cannot afford animal protein (Adeyemi et

\footnotetext{
* Corresponding author email: samfemmy2002@gmail.com
}

al., 2012). For human consumption, the cowpea is mainly grown for grain (dry and fresh) and sometimes for fresh pods in West Africa, India, and South America, while also grown for leaves in East Africa. It is an under used legume crop with a high potential for food and nutritional security in South Africa and produced for grain, immature green pods and fresh leaves due to its nutritional composition (Gerrano et al., 2015a; 2017a). The cowpea can be used to produce a large range of dishes and snacks (Uzogara and Ofuya, 1992; Asif et al., 2013). The consumption of the cowpea as a dietary staple in West Africa over millennia has produced extensive and varied culinary practices and many individual foods and dishes. Cowpea consumption in West Africa has led to a culinary practice that requires seed coat removal (also called decortication or dehulling). For example, the popular West African cowpea-based foods, such as Akara and Moin-moin, are decorticated (Phillips, 2012). The production and storage of cowpea have faced so many constraints, throughout West Africa 
such as diseases and the limited use of fertilizers and irrigation inputs (Brisibe et al., 2011) but major constraints is the insect pest known as Callosobruchus maculatus (Musa et al 2009), which infests it before and after harvest consequently leading to loss of economic value (Baidoo et al., 2010). Infestations on stored grains may reach $50 \%$ within 3-4 months of storage (Oparekeand Dike, 2005).

\section{Materials and Methods}

This study was conducted in the Department of Crop Protection laboratory and Ir. Leo Vande Mierop Biotechnology laboratory of the University of Ilorin, Ilorin, Nigeria.

\subsection{Source of cowpea seeds and plant materials}

The cowpea seeds used for the experiment were purchased from a local market (Oja-Oba) Ilorin, and the natural plant materials, Azadirachta indica (neem leaf), Vernonia amygdalina (Bitter leaf) and Carica papaya (pawpaw leaf) were sourced from the University of Ilorin premises.

\subsection{Insect Culture}

C. maculatus used for the experiment was obtained from Nigerian Stored Product Research Institute (NSPRI) Ilorin and this was used to establish a culture in the laboratory of the Department of Crop Protection. Freshly emerged dults of $C$. maculatus were used for the experiment

\subsection{Preparation of the Botanicals}

Fresh leaves of Neem, Bitter leaf and Pawpaw were collected and air dried for 7 days. The dried leaves were ground with mortar and pestle and sieved using $3 \mathrm{~mm}$ sieve to obtain a fine powder. The leaf powders were separately packed into air tight containers until required for use.

\subsection{Experimental Procedure}

The purchased cowpea seeds were disinfected by storing in a deep freezer for 72 hours at $4^{\circ} \mathrm{C}$ to kill any hidden $C$. maculatus in the seeds. $100 \mathrm{~g}$ of cowpea seeds were weighed and put into transparent plastic containers.

The plant powders were weighed and applied at the following rates; $3 \mathrm{~g}, 6 \mathrm{~g}$ and $9 \mathrm{~g}$ respectively. The containers were shaken to ensure uniform covering of the seeds with the treatments. 6 unsexed freshly emerged adult $C$. maculatus were introduced into each container and the container covered with muslin cloth held in place with the aid of a rubber band.

The experiment was carried out using a completely randomized design. There were 23 treatments and each treatment was replicated 4 times giving a total of 92 experimental units. In the mixed treatments, the powders were mixed in equal ratios. Cypermethrin was used as the positive controls at recommended dose $(0.6 \mathrm{~g} / 100 \mathrm{~g}$ of cowpea seeds).

\subsection{Data Collection}

Data were collected on the following parameters: adult mortality of the $C$. maculatus were carried out at 24hours, 3day, day 5, day 7 and day 9 after the treatment and then recorded (A beetle was assumed dead if there is no movement of its legs and antenna and also if it did not respond to a pin probe at its abdomen), larval emergence was taken at $15^{\text {th }}$ day and $17^{\text {th }}$ day post treatment, pupa emergence was taken at $17^{\text {th }}$ day and $19^{\text {th }}$ day post treatment. The larvae and pupae are normally only found in cells bored within the seeds of pulses. For descriptions and a key including $C$. maculatus larvae. The weight loss of the seeds was taken after the whole experiment

\subsection{Data Analysis}

The data collected were subjected to analysis of variance (ANOVA) and treatment means that were significantly different were separated using the New Duncan Multiple Range Test at $\mathrm{P}=0.05$ level of probability. 
Solihu et al. / Selcuk J Agr Food Sci, (2021) 35 (1), 49-55

Table 1

Effect of the treatments on percentage (\%) adult mortality of Callosobruchus maculatus

\begin{tabular}{|c|c|c|c|c|c|c|}
\hline \multirow[t]{2}{*}{ Treatment } & \multirow[t]{2}{*}{ Rate(g) } & \multicolumn{5}{|c|}{ Days after treatment(DAT) } \\
\hline & & 1 & 3 & 5 & 7 & 9 \\
\hline BL & 3 & $8.34 \pm 9.62 \mathrm{abc}$ & $25 \pm 9.62 \mathrm{bcde}$ & $20.83 \pm 15.96 a b$ & $21.08 \pm 15.52 \mathrm{ab}$ & $4.17 \pm 8.34 b c$ \\
\hline BL & 6 & $12.5 \pm 15.96 \mathrm{abc}$ & $33.33 \pm 0 \mathrm{abcd}$ & $16.67 \pm 13.61 \mathrm{ab}$ & $12.5 \pm 8.34 \mathrm{ab}$ & $16.67 \pm 13.61 \mathrm{abc}$ \\
\hline BL & 9 & $25 \pm 9.62 \mathrm{a}$ & $20.83 \pm 15.96 \mathrm{cde}$ & $12.5 \pm 8.34 \mathrm{ab}$ & $20.83 \pm 15.96 \mathrm{ab}$ & $12.5 \pm 8.34 \mathrm{abc}$ \\
\hline NL & 3 & $12.5 \pm 15.96 \mathrm{abc}$ & $29.17 \pm 8.33 \mathrm{abcde}$ & $16.67 \pm 23.57 \mathrm{ab}$ & $25 \pm 16.67 \mathrm{ab}$ & $12.5 \pm 15.96 \mathrm{abc}$ \\
\hline NL & 6 & $12.5 \pm 15.96 \mathrm{abc}$ & $29.17 \pm 15.96$ abcde & $20.84 \pm 8.33 \mathrm{ab}$ & $16.67 \pm 13.61 \mathrm{ab}$ & $20.84 \pm 8.33 \mathrm{ab}$ \\
\hline NL & 9 & $16.67 \pm 0 \mathrm{abc}$ & $20.84 \pm 8.33$ cde & $12.5 \pm 15.96 \mathrm{ab}$ & $25 \pm 21.52 \mathrm{ab}$ & $16.67 \pm 13.61 \mathrm{abc}$ \\
\hline PL & 3 & $16.67 \pm 0 \mathrm{abc}$ & $20.83 \pm 15.96 \mathrm{cde}$ & $33.33 \pm 13.61 \mathrm{a}$ & $16.67 \pm 13.61 \mathrm{ab}$ & $12.5 \pm 8.34 \mathrm{abc}$ \\
\hline PL & 6 & $16.67 \pm 13.61 \mathrm{abc}$ & $37.5 \pm 15.96 \mathrm{abcd}$ & $29.17 \pm 28.46 \mathrm{a}$ & $8.34 \pm 9.62 b$ & $4.17 \pm 8.34 \mathrm{bc}$ \\
\hline PL & 9 & $20.83 \pm 15.96 a b$ & $41.67 \pm 9.62 \mathrm{abcd}$ & $16.67 \pm 0 \mathrm{ab}$ & $12.5 \pm 15.96 \mathrm{ab}$ & $4.17 \pm 8.34 \mathrm{bc}$ \\
\hline BLNL & 3 & $4.17 \pm 8.34 \mathrm{bc}$ & $29.17 \pm 25 \mathrm{abcde}$ & $12.5 \pm 15.96 \mathrm{ab}$ & $33.34 \pm 23.57 \mathrm{a}$ & $12.5 \pm 15.96 \mathrm{abc}$ \\
\hline BLNL & 6 & $12.5 \pm 8.34 \mathrm{abc}$ & $50 \pm 0 \mathrm{a}$ & $16.67 \pm 0 \mathrm{ab}$ & $12.5 \pm 8.34 \mathrm{ab}$ & $4.17 \pm 8.34 b c$ \\
\hline BLNL & 9 & $4.17 \pm 8.34 \mathrm{bc}$ & $33.34 \pm 19.24 \mathrm{abcd}$ & $25 \pm 9.62 \mathrm{ab}$ & $16.67 \pm 0 \mathrm{ab}$ & $8.34 \pm 9.62 \mathrm{abc}$ \\
\hline NLPL & 3 & $4.17 \pm 8.34 \mathrm{bc}$ & $29.17 \pm 15.96 \mathrm{abcde}$ & $16.67 \pm 13.61 \mathrm{ab}$ & $20.83 \pm 15.96 \mathrm{ab}$ & $25 \pm 9.62 \mathrm{a}$ \\
\hline NLPL & 6 & $12.5 \pm 15.96 \mathrm{abc}$ & $16.67 \pm 0 \mathrm{de}$ & $33.33 \pm 23.57 \mathrm{a}$ & $25 \pm 9.62 \mathrm{ab}$ & $12.5 \pm 15.96 \mathrm{abc}$ \\
\hline NLPL & 9 & $4.17 \pm 8.34 \mathrm{bc}$ & $29.17 \pm 15.96 \mathrm{abcde}$ & $16.67 \pm 0 \mathrm{ab}$ & $25 \pm 9.62 \mathrm{ab}$ & $8.34 \pm 9.62 \mathrm{abc}$ \\
\hline BLPL & 3 & $8.34 \pm 9.62 \mathrm{abc}$ & $33.33 \pm 0 \mathrm{abcd}$ & $20.83 \pm 15.96 a b$ & $20.84 \pm 8.33 \mathrm{ab}$ & $4.17 \pm 8.34 b c$ \\
\hline BLPL & 6 & $8.34 \pm 9.62 \mathrm{abc}$ & $45.83 \pm 8.34 \mathrm{ab}$ & $29.17 \pm 8.33 a$ & $8.34 \pm 9.62 b$ & $0 \pm 0 \mathrm{c}$ \\
\hline BLPL & 9 & $0 \pm 0 \mathrm{c}$ & $37.5 \pm 8.33 \mathrm{abcd}$ & $29.17 \pm 8.33 \mathrm{a}$ & $25 \pm 9.62 \mathrm{ab}$ & $8.34 \pm 9.62 \mathrm{abc}$ \\
\hline BLNLPL & 3 & $16.67 \pm 0 \mathrm{abc}$ & $29.17 \pm 8.33 \mathrm{abcde}$ & $20.84 \pm 8.33 \mathrm{ab}$ & $16.67 \pm 0 \mathrm{ab}$ & $12.5 \pm 8.34 \mathrm{abc}$ \\
\hline BLNLPL & 6 & $12.5 \pm 8.34 \mathrm{abc}$ & $29.17 \pm 15.96 \mathrm{abcde}$ & $16.67 \pm 13.61 \mathrm{ab}$ & $20.84 \pm 8.33 \mathrm{ab}$ & $20.83 \pm 15.96 a b$ \\
\hline BLNLPL & 9 & $20.84 \pm 8.33 \mathrm{ab}$ & $25 \pm 16.67 \mathrm{bcde}$ & $12.5 \pm 8.34 \mathrm{ab}$ & $25 \pm 9.62 \mathrm{ab}$ & $16.67 \pm 13.61 \mathrm{abc}$ \\
\hline Cypermethrin & & $16.67 \pm 13.61 \mathrm{abc}$ & $33.34 \pm 19.24 \mathrm{abcd}$ & $33.33 \pm 13.61 \mathrm{a}$ & $16.67 \pm 0 \mathrm{ab}$ & $12.5 \pm 15.96 \mathrm{abc}$ \\
\hline Control & & $8.34 \pm 9.62 \mathrm{abc}$ & $8.34 \pm 9.62 \mathrm{e}$ & $4.17 \pm 8.34 \mathrm{~b}$ & $4.17 \pm 8.34 \mathrm{~b}$ & $8.34 \pm 9.62 b$ \\
\hline S.E.M & & 5.31 & 6.64 & 6.95 & 6.23 & 5.63 \\
\hline
\end{tabular}

Values with the same letter(s) in the same column are not significantly different $5 \%$ level of significance using Duncan's multiple range test

KEY: DAT = Days after Treatment, SEM=Standard error of mean, BL= Bitter leaf, NL=Neem leaf, PL=Pawpaw Leaf

Table 2

Effects of treatments on Larva and Pupa emergence

\begin{tabular}{|c|c|c|c|c|c|}
\hline \multirow[t]{3}{*}{ Treatment } & \multirow[t]{3}{*}{ Rate (g) } & \multicolumn{4}{|c|}{ Days after treatment (DAT) } \\
\hline & & \multicolumn{2}{|c|}{ Larva emergence } & \multicolumn{2}{|c|}{ Pupa emergence } \\
\hline & & 15 & 17 & 17 & 19 \\
\hline $\mathrm{BL}$ & 3 & $0 \pm 0 \mathrm{c}$ & $0 \pm 0 \mathrm{~b}$ & $0 \pm 0 b$ & $0.75 \pm 0.5 \mathrm{abc}$ \\
\hline BL & 6 & $0 \pm 0 \mathrm{c}$ & $0 \pm 0 \mathrm{~b}$ & $0.25 \pm 0.5 b$ & $1 \pm 1.15 \mathrm{abc}$ \\
\hline $\mathrm{BL}$ & 9 & $0 \pm 0 \mathrm{c}$ & $0 \pm 0 \mathrm{~b}$ & $0.5 \pm 0.58 \mathrm{ab}$ & $0.75 \pm 0.96 \mathrm{abc}$ \\
\hline NL & 3 & $0 \pm 0 \mathrm{c}$ & $0 \pm 0 \mathrm{~b}$ & $0 \pm 0 \mathrm{~b}$ & $0.25 \pm 0.5 \mathrm{c}$ \\
\hline NL & 6 & $0 \pm 0 \mathrm{abc}$ & $0 \pm 0 \mathrm{~b}$ & $0.5 \pm 0.58 \mathrm{ab}$ & $0 \pm 0 \mathrm{c}$ \\
\hline NL & 9 & $0 \pm 0 \mathrm{abc}$ & $0 \pm 0 \mathrm{~b}$ & $0.5 \pm 0.58 \mathrm{ab}$ & $1 \pm 0.82 \mathrm{abc}$ \\
\hline PL & 3 & $0 \pm 0 \mathrm{abc}$ & $0 \pm 0 \mathrm{~b}$ & $0 \pm 0 b$ & $1 \pm 0.82 \mathrm{abc}$ \\
\hline PL & 6 & $0 \pm 0 \mathrm{abc}$ & $0.5 \pm 0.58 \mathrm{ab}$ & $0 \pm 0 b$ & $0.75 \pm 0.5 \mathrm{abc}$ \\
\hline PL & 9 & $0.25 \pm 0.5 \mathrm{ab}$ & $0.75 \pm 0.5 \mathrm{a}$ & $0 \pm 0 \mathrm{~b}$ & $0.75 \pm 0.5 \mathrm{abc}$ \\
\hline BLNL & 3 & $0 \pm 0 \mathrm{c}$ & $0 \pm 0 \mathrm{~b}$ & $0 \pm 0 \mathrm{~b}$ & $0 \pm 0 \mathrm{c}$ \\
\hline
\end{tabular}


Table 2

Effects of treatments on Larva and Pupa emergence

\begin{tabular}{llllll}
\hline BLNL & 6 & $0 \pm 0 \mathrm{c}$ & $0 \pm 0 \mathrm{~b}$ & $0 \pm 0 \mathrm{~b}$ & $0.5 \pm 1 \mathrm{bc}$ \\
BLNL & 9 & $0 \pm 0 \mathrm{c}$ & $0 \pm 0 \mathrm{~b}$ & $0 \pm 0 \mathrm{~b}$ & $0.75 \pm 0.96 \mathrm{abc}$ \\
NLPL & 3 & $0 \pm 0 \mathrm{abc}$ & $0.25 \pm 0.5 \mathrm{ab}$ & $0.25 \pm 0.5 \mathrm{~b}$ & $0.75 \pm 0.96 \mathrm{abc}$ \\
NLPL & 6 & $0 \pm 0 \mathrm{abc}$ & $0.5 \pm 0.58 \mathrm{ab}$ & $0 \pm 0 \mathrm{~b}$ & $1.75 \pm 2.22 \mathrm{abc}$ \\
NLPL & 9 & $0 \pm 0 \mathrm{abc}$ & $0 \pm 0 \mathrm{~b}$ & $0 \pm 0 \mathrm{~b}$ & $1 \pm 1.15 \mathrm{abc}$ \\
BLPL & 3 & $0 \pm 0 \mathrm{c}$ & $0 \pm 0 \mathrm{~b}$ & $0 \pm 0 \mathrm{~b}$ & $1 \pm 0 \mathrm{abc}$ \\
BLPL & 6 & $0 \pm 0 \mathrm{c}$ & $0 \pm 0 \mathrm{~b}$ & $0 \pm 0 \mathrm{~b}$ & $0.5 \pm 0.58 \mathrm{bc}$ \\
BLPL & 9 & $0 \pm 0 \mathrm{c}$ & $0 \pm 0 \mathrm{~b}$ & $0.5 \pm 0.58 \mathrm{ab}$ & $1 \pm 0.82 \mathrm{abc}$ \\
BLNLPL & 3 & $0 \pm 0 \mathrm{c}$ & $0 \pm 0 \mathrm{~b}$ & $0 \pm 0 \mathrm{~b}$ & $0.25 \pm 0.5 \mathrm{c}$ \\
BLNLPL & 6 & $0 \pm 0 \mathrm{c}$ & $0.25 \pm 0.5 \mathrm{ab}$ & $0.25 \pm 0.5 \mathrm{~b}$ & $0.25 \pm 0.5 \mathrm{c}$ \\
BLNLPL & & $0 \pm 0 \mathrm{c}$ & $0.25 \pm 0.5 \mathrm{ab}$ & $0.5 \pm 0.58 \mathrm{ab}$ & $0.5 \pm 1 \mathrm{bc}$ \\
Cypermethrin & & $0 \pm 0 \mathrm{abc}$ & $0 \pm 0 \mathrm{~b}$ & $0 \pm 0 \mathrm{~b}$ & $2 \pm 0 \mathrm{a}$ \\
Control & & $0.25 \pm 0.5 \mathrm{a}$ & $0.75 \pm 0.96 \mathrm{a}$ & $1 \pm 0.82 \mathrm{a}$ & $0.5 \pm 0.58 \mathrm{bc}$ \\
S.E.M & & 0.0737 & 0.1676 & 0.1831 & 0.4235 \\
\hline
\end{tabular}

Values with the same letter(s) in the same column are not significantly different 5\% level of significance using Duncan's multiple range test

KEY: DAT = Days after Treatment, SEM=Standard error of mean, BL= Bitter leaf, NL=Neem leaf, PL=Pawpaw Leaf

Table $3 \mathrm{a}$

Effects of treatments on $\mathrm{F}_{1}$ progeny emergence of $C$. maculatus

\begin{tabular}{|c|c|c|c|c|c|c|}
\hline \multirow[t]{2}{*}{ Treatment } & \multirow[t]{2}{*}{ Rate(g) } & \multicolumn{5}{|c|}{ Days after treatment (DAT) } \\
\hline & & 28 & 30 & 32 & 34 & \\
\hline $\mathrm{BL}$ & 3 & $0.75 \pm 0.5 \mathrm{abc}$ & $1 \pm 1.15 \mathrm{ac}$ & $1 \pm 1.15 \mathrm{ab}$ & $2.5 \pm 1.91 \mathrm{a}$ & $\overline{0.5 \pm 1 b}$ \\
\hline BL & 6 & $1.25 \pm 0.5 \mathrm{abc}$ & $0.25 \pm 0.5 \mathrm{c}$ & $1 \pm 2 \mathrm{ab}$ & $2.5 \pm 3.7 \mathrm{a}$ & $0.5 \pm 0.58 b$ \\
\hline BL & 9 & $2 \pm 1.83 \mathrm{a}$ & $0.25 \pm 0.5 \mathrm{c}$ & $0.25 \pm 0.5 b$ & $1.75 \pm 2.06 \mathrm{a}$ & $0 \pm 0 \mathrm{~b}$ \\
\hline NL & 3 & $0.75 \pm 0.96 \mathrm{abc}$ & $0.5 \pm 1 \mathrm{c}$ & $1.25 \pm 2.5 \mathrm{ab}$ & $5.25 \pm 10.5 \mathrm{a}$ & $1.5 \pm 3 b$ \\
\hline NL & 6 & $0.5 \pm 0.58 \mathrm{abc}$ & $0 \pm 0 \mathrm{c}$ & $0.25 \pm 0.5 b$ & $0 \pm 0 \mathrm{a}$ & $0 \pm 0 \mathrm{~b}$ \\
\hline NL & 9 & $0.25 \pm 0.5 b c$ & $2.5 \pm 1 \mathrm{ab}$ & $1.25 \pm 0.5 \mathrm{ab}$ & $4.25 \pm 3.1 \mathrm{a}$ & $0.25 \pm 0.5 b$ \\
\hline PL & 3 & $0.5 \pm 0.58 \mathrm{abc}$ & $0.5 \pm 0.58 \mathrm{c}$ & $0.75 \pm 0.96 \mathrm{ab}$ & $2 \pm 2.71 \mathrm{a}$ & $0.75 \pm 0.96 b$ \\
\hline PL & 6 & $0.75 \pm 0.5 \mathrm{abc}$ & $0.75 \pm 0.5 \mathrm{c}$ & $3.25 \pm 5.19 \mathrm{a}$ & $10.75 \pm 21.5 a$ & $3.25 \pm 5.25 b$ \\
\hline PL & 9 & $1.5 \pm 1.29 \mathrm{abc}$ & $1 \pm 0.82 \mathrm{abc}$ & $0.5 \pm 0.58 b$ & $1.75 \pm 1.26 \mathrm{a}$ & $0 \pm 0 \mathrm{~b}$ \\
\hline BLNL & 3 & $1.5 \pm 1.29 \mathrm{abc}$ & $1 \pm 2 \mathrm{abc}$ & $0.75 \pm 0.96 \mathrm{ab}$ & $2 \pm 3.37 \mathrm{a}$ & $1.25 \pm 1.89 \mathrm{~b}$ \\
\hline BLNL & 6 & $0.5 \pm 0.58 \mathrm{abc}$ & $0 \pm 0 \mathrm{c}$ & $0.5 \pm 0.58 b$ & $0 \pm 0 \mathrm{a}$ & $0.25 \pm 0.5 b$ \\
\hline BLNL & 9 & $1.75 \pm 1.5 \mathrm{ab}$ & $0.25 \pm 0.5 c$ & $0.25 \pm 0.5 b$ & $1.25 \pm 1.26 \mathrm{a}$ & $0.75 \pm 0.96 b$ \\
\hline NLPL & 3 & $0.5 \pm 0.58 \mathrm{abc}$ & $0.5 \pm 1 \mathrm{c}$ & $0 \pm 0 \mathrm{~b}$ & $2.75 \pm 2.87 \mathrm{a}$ & $1.5 \pm 1.73 b$ \\
\hline NLPL & 6 & $1.5 \pm 1.73 \mathrm{abc}$ & $1 \pm 2 \mathrm{abc}$ & $1 \pm 2 \mathrm{ab}$ & $5.25 \pm 10.5 a$ & $2.75 \pm 3.77 \mathrm{~b}$ \\
\hline NLPL & 9 & $1.75 \pm 1.5 \mathrm{ab}$ & $0.5 \pm 0.58 \mathrm{c}$ & $1 \pm 0 \mathrm{ab}$ & $0.5 \pm 0.58 \mathrm{a}$ & $0 \pm 0 \mathrm{~b}$ \\
\hline BLPL & 3 & $1.5 \pm 1 \mathrm{abc}$ & $0 \pm 0 \mathrm{c}$ & $0.25 \pm 0.5 b$ & $0 \pm 0 \mathrm{a}$ & $0.75 \pm 1.5 b$ \\
\hline BLPL & 6 & $0.5 \pm 0.58 \mathrm{abc}$ & $0.5 \pm 1 \mathrm{c}$ & $1.5 \pm 1.73 \mathrm{ab}$ & $2.25 \pm 4.5 \mathrm{a}$ & $0 \pm 0 \mathrm{~b}$ \\
\hline BLPL & 9 & $1 \pm 0 \mathrm{abc}$ & $1 \pm 1.15 \mathrm{abc}$ & $0 \pm 0 \mathrm{~b}$ & $2.5 \pm 2.89 \mathrm{a}$ & $0.75 \pm 1.5 b$ \\
\hline BLNLPL & 3 & $0.5 \pm 0.58 \mathrm{abc}$ & $0.25 \pm 0.5 \mathrm{c}$ & $1.25 \pm 1.26 \mathrm{ab}$ & $1 \pm 0 \mathrm{a}$ & $1.25 \pm 2.5 b$ \\
\hline BLNLPL & 6 & $1.75 \pm 0.5 \mathrm{ab}$ & $0 \pm 0 \mathrm{c}$ & $0 \pm 0 \mathrm{~b}$ & $0.75 \pm 0.96 a$ & $0.25 \pm 0.5 b$ \\
\hline BLNLPL & 9 & $0 \pm 0 \mathrm{c}$ & $2.5 \pm 2.38 \mathrm{a}$ & $0.5 \pm 0.58 b$ & $5 \pm 4.55 \mathrm{a}$ & $1.25 \pm 2.5 b$ \\
\hline Cypermethrin & & $0 \pm 0 \mathrm{c}$ & $0.75 \pm 0.5 \mathrm{c}$ & $2.5 \pm 1 \mathrm{ab}$ & $7.25 \pm 7.23 a$ & $0.75 \pm 0.5 b$ \\
\hline Control & & $0.75 \pm 0.96 \mathrm{abc}$ & $1 \pm 0.82 \mathrm{abc}$ & $2 \pm 1.63 \mathrm{ab}$ & $8 \pm 6.63 \mathrm{a}$ & $7.25 \pm 2.99 a$ \\
\hline S.E.M & & 0.4692 & 0.509 & 0.772 & 3.112 & 0.975 \\
\hline
\end{tabular}

Values with the same letter(s) in the same column are not significantly different 5\% level of significance using Duncan's multiple range test

KEY: DAT = Days after Treatment, SEM=Standard error of mean, BL= Bitter leaf, NL=Neem leaf, PL=Pawpaw Leaf 
Table $3 b$

Effects of treatments on $\mathrm{F}_{1}$ progeny emergence of $C$. maculatus (continuation)

\begin{tabular}{|c|c|c|c|c|c|c|}
\hline \multirow[t]{2}{*}{ Treatment } & \multirow[t]{2}{*}{ Rate(g) } & \multicolumn{5}{|c|}{ Days after treatment (DAT) } \\
\hline & & 38 & 40 & 42 & 44 & 46 \\
\hline $\mathrm{BL}$ & 3 & $0.25 \pm 0.5 b c$ & $0.25 \pm 0.5 b$ & $0 \pm 0 \mathrm{a}$ & $0 \pm 0 \mathrm{~b}$ & $0 \pm 0 \mathrm{~b}$ \\
\hline BL & 6 & $0.5 \pm 0.58 b c$ & $1.25 \pm 2.5 \mathrm{ab}$ & $0 \pm 0 \mathrm{a}$ & $0 \pm 0 \mathrm{~b}$ & $0 \pm 0 \mathrm{~b}$ \\
\hline $\mathrm{BL}$ & 9 & $0 \pm 0 \mathrm{c}$ & $0 \pm 0 \mathrm{~b}$ & $0 \pm 0 \mathrm{a}$ & $0 \pm 0 \mathrm{~b}$ & $0 \pm 0 \mathrm{~b}$ \\
\hline NL & 3 & $1 \pm 2 b c$ & $0.75 \pm 1.5 b$ & $0.25 \pm 0.5 \mathrm{a}$ & $0.25 \pm 0.5 b$ & $0 \pm 0 \mathrm{~b}$ \\
\hline NL & 6 & $0 \pm 0 \mathrm{bc}$ & $0 \pm 0 \mathrm{~b}$ & $0 \pm 0 \mathrm{a}$ & $0 \pm 0 \mathrm{~b}$ & $0 \pm 0 \mathrm{~b}$ \\
\hline NL & 9 & $0.25 \pm 0.5 \mathrm{bc}$ & $0.5 \pm 0.58 b$ & $0 \pm 0 \mathrm{a}$ & $0 \pm 0 \mathrm{~b}$ & $0 \pm 0 \mathrm{~b}$ \\
\hline PL & 3 & $0.75 \pm 0.96 b c$ & $0.5 \pm 0.58 b$ & $0 \pm 0 \mathrm{a}$ & $1.5 \pm 3 b$ & $0 \pm 0 b$ \\
\hline PL & 6 & $2.25 \pm 3.86 b$ & $1.25 \pm 2.5 \mathrm{ab}$ & $0.75 \pm 1.5 \mathrm{a}$ & $0.5 \pm 1 b$ & $0 \pm 0 \mathrm{~b}$ \\
\hline PL & 9 & $0 \pm 0 \mathrm{bc}$ & $0.25 \pm 0.5 b$ & $0 \pm 0 \mathrm{a}$ & $0 \pm 0 \mathrm{~b}$ & $0 \pm 0 \mathrm{~b}$ \\
\hline BLNL & 3 & $1 \pm 0.82 \mathrm{bc}$ & $0 \pm 0 \mathrm{~b}$ & $0 \pm 0 \mathrm{a}$ & $0 \pm 0 \mathrm{~b}$ & $0 \pm 0 \mathrm{~b}$ \\
\hline BLNL & 6 & $0.25 \pm 0.5 b c$ & $0.25 \pm 0.5 b$ & $0 \pm 0 \mathrm{a}$ & $0 \pm 0 \mathrm{~b}$ & $0 \pm 0 \mathrm{~b}$ \\
\hline BLNL & 9 & $0 \pm 0 \mathrm{c}$ & $0 \pm 0 \mathrm{~b}$ & $0 \pm 0 \mathrm{a}$ & $0 \pm 0 \mathrm{~b}$ & $0 \pm 0 \mathrm{~b}$ \\
\hline NLPL & 3 & $1.5 \pm 1 b c$ & $0 \pm 0 \mathrm{~b}$ & $0 \pm 0 \mathrm{a}$ & $0 \pm 0 \mathrm{~b}$ & $0 \pm 0 \mathrm{~b}$ \\
\hline NLPL & 6 & $1.5 \pm 3 b c$ & $0.5 \pm 1 b$ & $1.25 \pm 2.5 \mathrm{a}$ & $1.25 \pm 2.5 b$ & $0.5 \pm 1 b$ \\
\hline NLPL & 9 & $0 \pm 0 \mathrm{bc}$ & $0 \pm 0 \mathrm{~b}$ & $0 \pm 0 \mathrm{a}$ & $0 \pm 0 \mathrm{~b}$ & $0 \pm 0 \mathrm{~b}$ \\
\hline BLPL & 3 & $0.25 \pm 0.5 b c$ & $0 \pm 0 \mathrm{~b}$ & $0 \pm 0 \mathrm{a}$ & $0 \pm 0 \mathrm{~b}$ & $0 \pm 0 \mathrm{~b}$ \\
\hline BLPL & 6 & $0.25 \pm 0.5 b c$ & $0 \pm 0 \mathrm{~b}$ & $0 \pm 0 \mathrm{a}$ & $0 \pm 0 \mathrm{~b}$ & $0 \pm 0 \mathrm{~b}$ \\
\hline BLPL & 9 & $0.25 \pm 0.5 b c$ & $0 \pm 0 \mathrm{~b}$ & $0 \pm 0 \mathrm{a}$ & $0 \pm 0 \mathrm{~b}$ & $0 \pm 0 \mathrm{~b}$ \\
\hline BLNLPL & 3 & $0.5 \pm 1 b c$ & $0.5 \pm 1 b$ & $0 \pm 0 \mathrm{a}$ & $0 \pm 0 \mathrm{~b}$ & $0 \pm 0 \mathrm{~b}$ \\
\hline BLNLPL & 6 & $0 \pm 0 \mathrm{bc}$ & $0 \pm 0 \mathrm{~b}$ & $0 \pm 0 \mathrm{a}$ & $0 \pm 0 \mathrm{~b}$ & $0 \pm 0 \mathrm{~b}$ \\
\hline BLNLPL & 9 & $0.75 \pm 1.5 \mathrm{bc}$ & $1.25 \pm 2.5 \mathrm{ab}$ & $1.25 \pm 2.5 \mathrm{a}$ & $1.25 \pm 2.5 b$ & $2.75 \pm 5.5 \mathrm{a}$ \\
\hline Cypermethrin & & $0.5 \pm 1 b c$ & $3 \pm 3.46 a$ & $0 \pm 0 \mathrm{a}$ & $0 \pm 0 \mathrm{~b}$ & $0 \pm 0 \mathrm{~b}$ \\
\hline Control & & $6 \pm 1.63 \mathrm{a}$ & $2.25 \pm 2.87 \mathrm{ab}$ & $1.25 \pm 1.26 \mathrm{a}$ & $5.75 \pm 4.65 \mathrm{a}$ & $0 \pm 0 \mathrm{~b}$ \\
\hline S.E.M & & 0.653 & 0.697 & 0.4246 & 0.694 & 0.583 \\
\hline
\end{tabular}

Values with the same letter(s) in the same column are not significantly different $5 \%$ level of significance using Duncan's multiple range test

KEY: DAT = Days after Treatment, SEM=Standard error of mean, BL= Bitter leaf, NL=Neem leaf, PL=Pawpaw Leaf

Table 4

Effects of treatments on mean seed weight loss

\begin{tabular}{ccc}
\hline Treatment & Rate & Weight Loss $(\mathrm{g})$ \\
\hline BL & 3 & $0.48 \pm 0.43 \mathrm{c}$ \\
BL & 6 & $2.68 \pm 0.63 \mathrm{ab}$ \\
BL & 9 & $1.7 \pm 0.54 \mathrm{abc}$ \\
NL & 3 & $2.33 \pm 2.24 \mathrm{ab}$ \\
NL & 6 & $1.93 \pm 0.48 \mathrm{abc}$ \\
NL & 9 & $2.43 \pm 0.5 \mathrm{ab}$ \\
PL & 3 & $1.98 \pm 1.09 \mathrm{abc}$ \\
PL & 6 & $2 \pm 0.66 \mathrm{abc}$ \\
PL & 9 & $2.45 \pm 1.57 \mathrm{ab}$ \\
BLNL & 3 & $1.48 \pm 0.43 \mathrm{abc}$ \\
BLNL & 6 & $2.75 \pm 0.64 \mathrm{ab}$ \\
BLNL & 9 & $1.88 \pm 0.87 \mathrm{abc}$ \\
NLPL & 3 & $2.05 \pm 1.52 \mathrm{abc}$ \\
NLPL & 6 & $1.03 \pm 0.62 \mathrm{abc}$ \\
NLPL & 9 & $2.73 \pm 0.79 \mathrm{ab}$ \\
BLPL & 3 & $1.8 \pm 1.37 \mathrm{abc}$ \\
BLPL & 6 & $1.95 \pm 0.57 \mathrm{abc}$ \\
BLPL & 9 & $2.7 \pm 0.37 \mathrm{ab}$ \\
BLNLPL & 3 & $1.83 \pm 0.88 \mathrm{abc}$ \\
BLNLPL & 6 & $1.55 \pm 1.99 \mathrm{abc}$ \\
BLNLPL & 9 & $1.55 \pm 0.26 \mathrm{abc}$ \\
Cypermethrin & & $1.48 \pm 1.13 \mathrm{abc}$ \\
Control & & $2.93 \pm 0.38 \mathrm{a}$ \\
S.E.M & & 0.508 \\
\hline
\end{tabular}

Values with the same letter(s) in the same column are not significantly different $5 \%$ level of significance using Duncan's multiple range test

KEY: DAT = Days after Treatment, SEM=Standard error of mean, $\mathrm{BL}=$ Bitter leaf, $\mathrm{NL}=$ Neem leaf, $\mathrm{PL}=$ Pawpaw Leaf

\section{Results and Discussion}

The results of the study revealed that the various treatments used in the experiment had significant effects, mortality increased with increase in level of treatment. The plants leaf powder caused adult mortality of $C$. maculatus at the high and low rates when compared to the control, which was indicative of bioactive characteristics of the plant part. This is in agreement with the report of (Malungu et al., 2007) that the use of plant powders has been reported to produce higher death of insects because of physical barrier with the tendency of blocking the spiracles of the insects, thus impairing respiration leading to death of the insects. BLNL have the highest mortality followed by NLPL. Please cross check to confirm this claim by me. So with the mortality known that will affect the other experiment such as pupa emergence and weight loss. 
The insecticidal activity of powders of Vernonia amygdalina, Carica papaya and Azadirachta indica on larva and pupa emergence of adult Callosobruchus maculatus at different Days After Treatment (DAT) shows that there was a significant difference between the treatments and the control for larva and pupa emergence (Table 2 and 3). This could be attributed to the adult mortality already observed (Table 1) and the inhibition of oviposition as well as the remarkably high reduction in survival to adulthood of mature stages of C. maculatus compared to the control. This result corroborates that of (Okonkwo and Ewete 1999) in pepper fruit, (Babatunde and Musa, 2020) in Eucalyptus globulus leaf extract on cowpea beetle

The plants leaf powder was also observed to have effects in reducing the damage on cowpea seeds by $C$. maculatus (Table 4). Damage on cowpea seeds may have been reduced as a result of the extracts acting as a deterrent to $C$. maculatus, keeping them from infesting and damaging the seeds.

The study reveals that Vernonia amygdalina, Carica papaya and Azadirachta indica leaf powder could be very effective for use as bio-pesticides for protecting cowpea seeds from $C$. maculatus infestation and damage. It has been reported by the pest management specialists that botanicals are not known to leave any residue in any crop they are used to protect and the protective ability of essential oils could be attributed to interspecific insect responses to oil constituents (Enan, 2001).

The use of natural toxicants from plants as insecticides had been inexistent since the ancient times (Adebayo and Gbolade 1994 and Ismam, 2008, Babatunde et al 2020). The natural insecticides which require low cost to prepare, are readily available, environmentally and ecologically friendly are best suited for use in the storage of produce. (Babatund and Musa, 2020).

\section{Conclusion}

Plant extracts can be another source of pesticides against stored grain pests. It is recommended that the active molecule in Vernonia amygdalina, Carica papaya and Azadirachta indica responsible for their activities be isolated for the development of bio pesticides to protect grains in storage. For more effectiveness of plant extracts, a large amount proportional to the quantity of grains is required for post-harvest control of $C$. maculatus in stored cowpea for planting. This study has revealed that Vernonia amygdalina, Carica papaya and Azadirachta indica extract can be used to protect cowpea grains under small scale storage.

\section{References}

Abebe G, Hattar B, At-tawah A (2005). Nutrient Availability as Affected by Manure Application to Cowpea (Vigna Unguiculata L. Walp.) on Calacarious Soils. Journal of Agric. Soc. Sci., 1:1-6.

Adeyemi SA, Lewu FB, Adebola PO, Bradley G, Okoh A I (2012). Protein Content Variation in Cowpea Genotypes (Vigna Unguiculata L. Walp.) Grown in The Eastern Cape Province of South Africa as Affected by Mineralised Goat Manure. African Journal of Agricultural Research, 7(35):4943-4947.

Asif M (2013). "Application and opportunities of pulses in food system: A review", Critical Reviews in Food Science and Nutrition, Vol. 53, pp. 1168-1179.

Babatunde SF, Musa AK (2020). Effect of Tasmanian blue gum (Eucalyptus globulus Labill.) leaf extract on cowpea weevil (Callosobruchus maculatus [Fabricius, 1775], Coleoptera: Chrysomelidae). ActaagriculturaeSlovenica, 116/2, 351-356

Babatunde SF, Musa AK., Suleiman AF, Gambari LI (2021).Effect of cashew (Anacardium occidentale) nut shell stored and fresh extracts on cowpea bruchid, Callosobruchus maculatus (Fabricius.) (Coleoptera: Chrysomelidae). Acta fytotechnica et Zootechnica

Baidoo PK, Mochiah MB, Owusu-Akyaw M (2010). The Effect of Time of Harvest on the Damage Caused by the Cowpea Weevil Callosobruchus maculatus (Fab.) (Coleoptera: Bruchidae) Journal of Stored Products and Postharvest Research, 1(3):24 -28.

Brisibe EA, Adugbo SE, Ekanem U, Brisibe F, Figueira G M (2011). Controlling Bruchid Pests of Stored Cowpea Seeds with Dried Leaves of Artemisia annua and Two Other Common Botanicals. African Journal of Biotechnology, 10(47): 9586-9592 Center, Abidjan, Côte d'Ioire. pp: 367-375.

Dugje LO, Omoigui LO, Ekeleme F, Kamara AY, Ajeigbe H (2009). Farmers' Guide to Cowpea Production in West Africa. IITA, Ibadan, Nigeria. P 3. Ekesi S, Dike MS, Ogunlana MO (1996). Relationship between planting dates and damage by the legume pod-borer, Maruca testularis (Geyes) (Lepidoptera: Pyralidae) on cowpea, (Vigna unguiculata) L. (Walp.) in Nigeria. InternationalJournal of Pest Management. 42:315316.

Enan E (2001) Insecticidal activity of essential oils: octop-aminergic sites of action. Comparative Biochemistry and Physiology 130:325-337

Adebayo TA, Gbolade AA (1994). Protection of stored cowpea from Callosobruchus maculatus using plant products. Insect Sci. Applic. 15, 185-189.

Gerrano AS (2015a). "Genetic variability and heritability estimates of nutritional composition in 
the leaves of selected cowpea genotypes Vigna unguiculata (L.) Walp.", HortScience, Vol. 50(10), pp. 1435-1440.

Gerrano AS, Jansen van Rensburg WS, Adebola PO (2017a). "Preliminary evaluation of seed and germination traits in cowpea Vigna unguiculata (L.) Walp. genotypes", South African Journal of Plant and Soil, Vol.34(5), pp. 399-

402, https://doi.org/10.1080/02571862.2017.13178 49.

Ghaly A, Alkoaik F (2010). Effect of Municipal Solid Waste Compost on the Growth and Production of Vegetable Crops. American Journal of Agricultural and Biological Sciences, 5(3), 274-281. https://doi.org/10.3844/ajabssp.2010.274.281

Isman MB (2008). Botanical insecticides: for richer, for poorer. Pest management Science 64: 8-11.

Mulungu LS, Lupenza G, Reuben SO, Misangu RN (2007). Evaluation of Btoanical Products as Stored Grain Protectant against Cowpea weevil Callosobruchus maculatus. Journal of Entomology. 4(3): 258 - 349 .

Musa AM, Aliyu AB, Yaro AH, Magaji MG, Hassan HS, Abdullahi MI (2009). Preliminary phytochemical, analgesic and anti-inflammatory studies of the methanol extract of Anisopus Mannii (N.E.Br) (Asclepiadaceae) in rodents. African
Journal Pharmacy and Pharmacology. 3(8): 374378.

Okonkwo EU, Ewete MA (1999) Insecticidal effect of some spices on Sitophilus oryzae in rice. Int. J. Pest Manage; 41:143-145.

Oparaeke A, Dike MC (2005). Monodora myristica (Gaertn) Dunal. (Myristicaceae) and Allium Cepa L. (Liliaceae) as Protectants against Cowpea Seed Bruchid, Callosobruchus maculatus Infesting Stored Cowpea Seeds. NigerianJournal of Entomology, 22: 84-92.

Phillips RD (2012). "Cowpea Processing and Products", in Dry Beans and Pulses Production, Processing and Nutrition, Blackwell Publishing Ltd., pp. 235259, http://onlinelibrary.wiley.com/doi/10.1002/978 1118448298.ch 10

Singh BB (2014). "Cowpea: the food legume of the $21^{\text {st }}$ century" in Cowpea: The Food Legume of the $21^{\text {st }}$ Century, Crop Science Society of America ed., ACSESS Publication

Uzogara SG, Ofuya ZM (1992). Processing and tilization of cowpeas in developing countries - A review', Journal of Food Processing and Preservation, Vol. 16 pp. 105-147.

Xiong H, Shi A, Mou B, Qin J, Motes, Lu W (2016)

Genetic diversity and population structure of Cowpea (Vigna unguiculata L. Walp). PLoS ONE

11(8):e0160941. Doi:10.1371/Journal.pone.0160941 